Review

\title{
NONRATT021972 long-noncoding RNA: A promising IncRNA in diabetes-related diseases
}

\author{
Abhishek Suwal ${ }^{1 *}$, Ji-long Hao ${ }^{*}$, Xiu-fen Liu ${ }^{*}$, Dan-dan Zhou ${ }^{2 *}$, Om Prakash Pant ${ }^{1 *}$, Ying Gao ${ }^{3^{*}}$, Peng Hui ${ }^{1}$, \\ Xin-xuan Dai ${ }^{1}$, Cheng-wei Lu${ }^{1 凶}$ \\ 1. Department of Ophthalmology, The First Hospital of Jilin University, No. 71 of Xinmin St., Changchun, Jilin Province, 130021, China. \\ 2. Department of Radiology, The First Hospital of Jilin University, No. 71 of Xinmin St., Changchun, Jilin Province, 130021, China \\ 3. Department of Endocrinology, The First Hospital of Jilin University, No. 71 of xinmin St., Changchun, Jilin Province, 130021, China \\ * These authors contributed equally to this work. \\ $\square$ Corresponding author: Cheng-wei Lu, M.D., Ph.D., Department of Ophthalmology, the First Hospital of Jilin University, No. 71 of Xinmin St., Changchun, \\ Jilin Province, 130021, China. Email address: 1cwchina800@sina.com Telephone No: +8618684317115 \\ (c) Ivyspring International Publisher. This is an open access article distributed under the terms of the Creative Commons Attribution (CC BY-NC) license \\ (https://creativecommons.org/licenses/by-nc/4.0/). See http://ivyspring.com/terms for full terms and conditions.
}

Received: 2019.02.18; Accepted: 2019.05.02; Published: 2019.06.02

\begin{abstract}
Diabetes mellitus (DM) is a principal health problem with increasing incidence worldwide. It can be associated with various systemic diseases. Long non-coding RNA (IncRNA), a member of non-coding RNA has been newly linked with various human diseases. Recent evidence from animal experiments has shown that the incidence and development of type 2 diabetes are contributed by the atypical expression of IncRNA in which the biomarker with capable clinical potential was IncRNA NONRATT021972. In this review, we demonstrated the numerous functions of NONRATT021972 in different diabetes-related diseases including diabetic neuropathy, diabetic cardiac autonomic neuropathy, myocardial ischemia, and hepatic glucokinase dysfunction. The emerging evidence shows that the role of NONRATT021972 in diabetic-related disease is novel and therapeutic. These results direct us to conclude that NONRATT021972 is a potential diagnostic and future targeted therapy for diabetes-associated diseases.
\end{abstract}

Key words: Diabetes mellitus, diabetes-related diseases, long non-coding RNA, NONRATT021972, myocardial ischemia, hepatic glucokinase

\section{Introduction}

Long noncoding RNA (LncRNA), a member of non-coding RNA, is composed of transcripts which are more than 200 nucleotides in size [1, 2]. LncRNA are transliterated from either strand and categorized as sense, antisense, bidirectional, intergenic, or intronic in relation with the adjacent protein-coding genes [1]. The eukaryotic transcriptome is constituted of both a large numbers of noncoding RNAs (ncRNAs) as well as large set of protein-coding RNAs [3]. LncRNA produces a multifaceted controlling network via connections with co-activators, transcription factors and/or repressors that can inspire multiple facets of gene transcription [4]. Numerous researches have been performed to investigate the impact of lncRNA in numerous pathological and physiological circumstances. Trials of knocking out of some lncRNA in rats gave rise to atypical functions [4]. LncRNA are also engaged in the pathological progressions of various malignancies, cardiovascular and nervous system diseases [5-7]. Interestingly, the functions of lncRNA are initiated to be triggered after the former occasion which may be toxic stimuli or a stimulus yet to be discovered.

Diabetes mellitus (DM) is a principal health problem with 422 million adults suffering from diabetes in 2014 around the world [8]. It is described by less or absence of insulin production in the body. Type 2 DM (T2DM) is most abundant form of diabetes, approximately with more than $90 \%$ of all cases [9]. In a medical situation, neuropathic pain is the most common chronic complications in patient with T2DM which is characterized by typical symptoms of pathological pain including spontaneous pain and specific allodynia [10]. Recent evidence from animal experiments has shown that the 
incidence and development of type 2 diabetes is contributed by the atypical expression of lncRNA [11, 12], in which the biomarker with capable clinical potential was lncRNA NONRATT021972 [13].

NONRATT021972 is an lncRNA which was verified with a diabetes - encouraging effect whose sequence was satisfactorily determined [14]. Animal experiments have revealed that regulation of NONRATT021972 was amplified in diabetic rats, and contributed in the transmission of nociceptive signalling, particularly in neuropathic pain $[4,15,16]$. There are some data signifying that NONRATT021972 silencing helped to reduce inflammation, however, no thorough mechanisms were discovered in the field of inflammation even after knowing that NONRATT021972 could regulate $\mathrm{P}_{2} \mathrm{X}_{7}$ and $\mathrm{P} 2 \mathrm{X}_{3}$ receptors in dorsal root ganglia (DRG) [13]. In clinical application, there are no reports about how the disease of diabetes are influenced by NONRATT021972, and it is also unclear whether NONRATT021972 could be a predictor for T2DM in the clinical scenario but recent advances in this field has positive results for the association of NONRATT021972 with diabetes as well as cell biology [17-19]. Interestingly, bioinformatics data showed that NONRATT021972 influenced tumor necrosis factor- $a$ (TNF- $a$ ), and TNF- $a$ was a significant factor in the development of inflammation [20].

Further tests displayed that there existed a crosstalk between p38 mitogen activated protein kinase (p38 MAPK) signalling pathway and NONRATT021972. Inhibition of p38 MAPK signalling reduced NONRATT021972-induced expression of the $\mathrm{P}_{2} \mathrm{X}_{7}$ receptor and $\left[\mathrm{Ca}^{2+}\right] \mathrm{i}$ increment upon $\mathrm{P} 2 \mathrm{X}_{7}$ receptor activation [21]. The roles of lncRNA in the function of sympathetic nerves continue to be poorly considered. NONRATT021972 was noticed to be up-regulated in the diabetic rat's nervous system cells suggesting that NONRATT021972 may contribute in the pathophysiologic processes associated to the sympathetic neuron within the setting of diabetes [14]. In this review, we focus on the complications and disease associated with diabetes such as neuropathic pain, cardiac autonomic neuropathy, myocardial ischemia and the role of hepatic glucokinase on diabetes and the biological process of NONRATT021972 (Table 1).

\section{Diabetes associated diseases}

\section{Diabetic neuropathy (DNP)}

Nerve damage secondary to increased blood sugar level is DNP, mostly affecting peripheral nerves of legs and feet. It is the major reason accounting for peripheral neuropathy [22] and has characteristic symptoms of nerve pathological pain, including spontaneous pain, hyperalgesia, and allodynia.

Symptoms of DNP can vary from pain and numbness in legs and feet to problems with the cardiovascular system, blood vessels, genitourinary system, and gastrointestinal system, depending on the affected nerves. Usually, DNP has less severe symptoms but may be quite painful and disabling. Intractable pain induced by DM has become a sustainable problem in the field of pain therapy.

Peng et al. suggested that expression level of NONRATT021972 in DRG was highly expressed in the T2DM rat model [23]. The concentration of NONRATT021972 in T2DM patient serum was also higher compared to control healthy subjects. The levels of TNF-a and interleukin-6 (IL-6) increased in T2DM rats [21] and with the help of lncRNA NONRATT021972 small interference RNA (siRNA), the diabetes complicated with nervous inflammatory diseases were reduced [13]. Studies by Liu et al. showed that the $\mathrm{P} 2 \mathrm{X}_{7}$ receptor in DRG exhibit a vital role in DNP [19, 24]. NONRATT021972 siRNA treatment lessened the expression levels of $\mathrm{P}^{2} \mathrm{X}_{7}$ mRNA as well as protein and the activation of Satellite Glial Cells (SGC) in the DRG of T2DM rats (Figure 1). Furthermore, the NONRATT021972 siRNA treatment reduced the release of TNF- $\alpha$, thereby inhibiting the excitability of DRG neurons and decreasing the mechanical and thermal hyperalgesia in T2DM rats [19]. These findings highlight the importance of NONRATT021972 in DNP.

Table 1. NONRATT021972 influence in diabetes-associated diseases

\begin{tabular}{|c|c|c|c|c|c|c|}
\hline \multicolumn{2}{|l|}{ Diseases } & Related molecules & Expression & Study model & Tissues Involved & References \\
\hline \multicolumn{2}{|l|}{ DNP } & $\begin{array}{l}\text { TNF- } \alpha, \text { IL-6, GFAP, mRNA, P2X } \\
\text { P2 }_{3} \text {, ERK1/2 }\end{array}$ & Up-regulated & $\begin{array}{l}\text { rat and } \\
\text { human }\end{array}$ & $\begin{array}{l}\text { Dorsal root ganglia, HEK293 } \\
\text { cells, satellite glial cells }\end{array}$ & $\begin{array}{l}{[13,19,21,} \\
23]\end{array}$ \\
\hline \multicolumn{2}{|l|}{ DCAN } & TNF-a, IL-6, IRS-1 & Up-regulated & rat & $\begin{array}{l}\text { Dorsal root ganglia, superior } \\
\text { cervical ganglia }\end{array}$ & {$[18,21]$} \\
\hline \multirow[t]{2}{*}{$\begin{array}{l}\text { Cardiac function } \\
\text { after MI }\end{array}$} & $\begin{array}{l}\text { Pathophysiological process in SG } \\
\text { after MI }\end{array}$ & TNF-a, IL-6, p-38 MAPK, P2X & Up-regulated & rat & Stellate ganglia & {$[31-34]$} \\
\hline & $\begin{array}{l}\text { Abnormal changes in cardiac } \\
\text { function after MI }\end{array}$ & $\begin{array}{l}\text { p-ERK1/2, Protein, } \mathrm{P} \mathrm{X}_{7} \\
\text { TH/GAP43,HEK293 cell }\end{array}$ & & $\begin{array}{l}\text { rat and } \\
\text { human }\end{array}$ & $\begin{array}{l}\text { Superius cervical ganglia, } \\
\text { HEK293 cells }\end{array}$ & \\
\hline \multicolumn{2}{|c|}{ Sympathetic Neuronal injury after MI } & $\mathrm{P} 2 \mathrm{X}_{7}$, Protein, & Up-regulated & human & PC12 cell, HEK293 cell & {$[35,36]$} \\
\hline \multicolumn{2}{|c|}{ Hepatic glucokinase in T2 DM } & Hepatic glucokinase, GSK- $3 \beta$ & Up-regulated & rat & liver & {$[38,41,45]$} \\
\hline
\end{tabular}




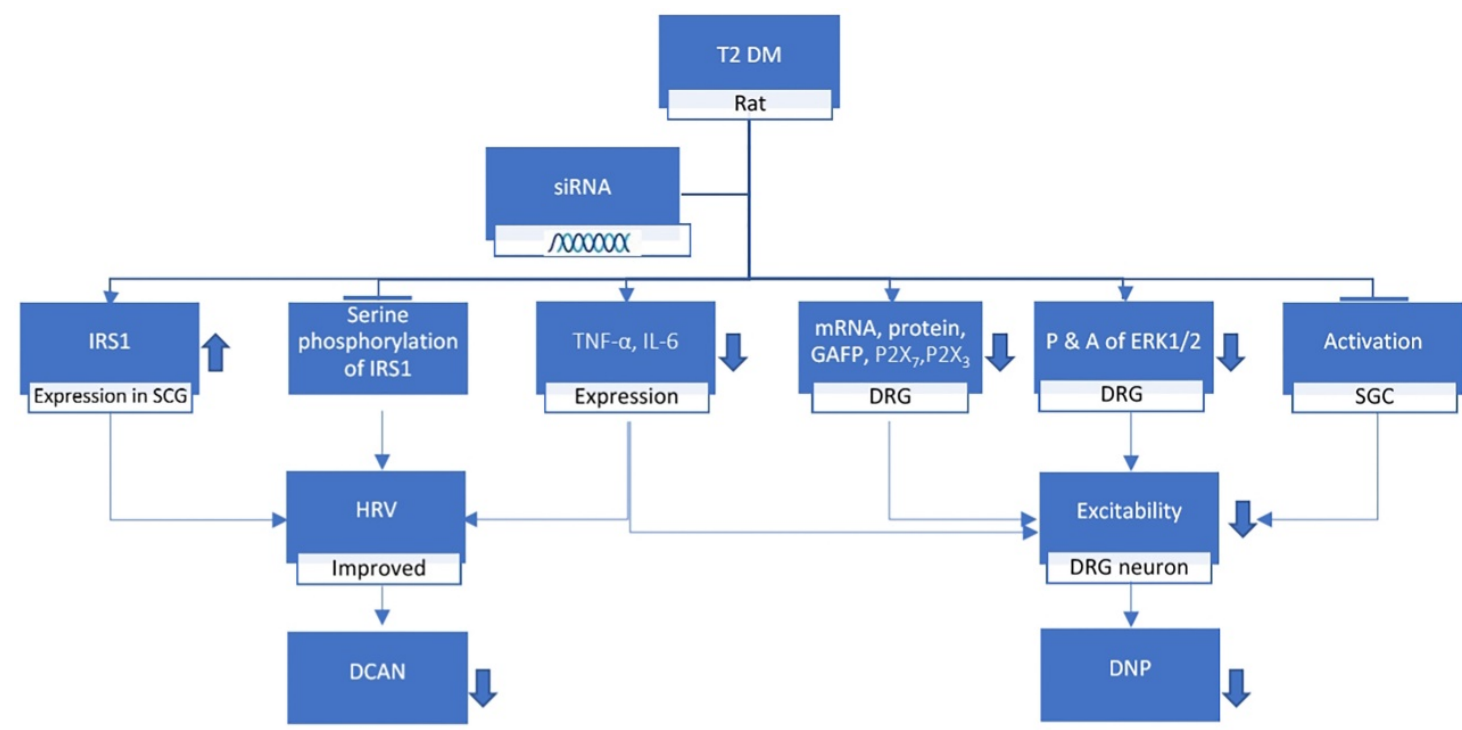

Figure 1. T2 DM, when treated with IncRNA NONRATT021972 siRNA, increases the insulin receptor substrate (IRS) 1 expression in superior cervical ganglia (SCG) whereas decreases the tumor necrosis factor- $\alpha$ (TNF- $\alpha$ ), interleukin-6 (IL-6), mRNA, P2 $X_{7}$, P2X $_{3}$, GAFP, ERK1/2 expression in Dorsal Root Ganglion (DRG) while inhibiting the serine phosphorylation of IRSI and activation of SGCs in the DRG. These results in the improvement of heart rate variable (HRV) while decreasing the excitability of DRG neuron which ultimately leads to the reduction of diabetic neuropathy and diabetic cardiac autonomic neuropathy.

\section{Diabetic cardiac autonomic neuropathy (DCAN)}

Diabetic autonomic neuropathy affecting the sympathetic and parasympathetic nervous systems is followed by cardiac autonomic dysfunction which is shown by impaired heart rate variability (HRV) [25]. Cardiac autonomic neuropathy is a common but rarely diagnosed complication of DM. It has a strong effect on various cardiac disorders including myocardial infarction and ischemia, high blood pressure, orthostatic hypotonia, heart failure, and arrhythmias [26].

Diabetic patients have two to four times higher incidence of cerebrovascular and cardiac disease than the general population [27]. Coronary artery disease (CAD) is the leading cause of morbidity and mortality in patients with DM. The impact of diabetes on CAD is synergistic with other factors, such as age, hypercholesterolemia, hypertension, and smoking.

It was found that there is an increase in NONRATT021972 in SCG of DM rats [18]. NONRATT021972's expression in sympathetic neuronal-like PC12 cells was found to be enhanced in the environmental setting of high glucose (HG) and high FFAs (HFs). Its knockdown expressively decreased HGHFs-induced TNF-a release in PC12 cells [21]. SCG has shown integrated effect on autonomic function in combination with the transmission of sympathetic preganglionic signals [28]. $\mathrm{Xu}$ et al. study on DM rat showed that NONRATT021972 expression was enhanced in sympathetic neuronal-like PC12 cells in the setting of HG and HFs [18]. To sum up, their study revealed that the over-expression of SCG TNF-a, blocked serine phosphorylation of IRS1 and enhanced IRS1 expression in SCG was inhibited by NONRATT021972 siRNA leading to improvement of HRV in diabetes (Figure 1). However extensive studies are needed in the field of NONRATT021972. This finding provides an innovative explanation for the universal role of the emergence of lncRNAs against neuroinflammation, which may be helpful for deepening our understanding of the related mechanism involved in the pathogenesis of DCAN. Thus, NONRATT021972 might prove to be a novel and efficacious bio-factor for DCAN.

\section{Cardiac function after myocardial ischemia}

Myocardial infarction (MI) is a life-threatening condition that occurs when blood flow to the heart muscle is shortly cut off resulting in tissue damage. If the flow of blood isn't restored quickly, heart muscle starts to die. Ischemic heart disease is one of the leading causes of death in economically developed countries worldwide [29]. Epidemiological studies and clinical trials have clearly shown that developing ischemic heart disease, including acute MI and postinfarct complications are common with both type 1 (insulin-dependent) and type 2 (noninsulindependent) DM [30].

MI ultimately leads to various cardiac dysfunctions. Studies from a rat experiment by Gao et al. suggested that there was an increase in CK-MB and $\mathrm{LDH}$ whereas the mean arterial blood pressure (MAP) was decreased [31]. Another study on humans gave us a clear understanding that the ageing heart was prone to myocardial ischemia where there were 
changes in the systolic blood pressure and diastolic blood pressure [32]. The dysregulated expression of lncRNAs participated in the onset and progression of several pathological conditions [33]. Experiments on rats showed that NONRATT021972 expression in stellate ganglia (SG) was significantly higher in the MI group than in the control group [34]. Increased systolic blood pressure (SBP), diastolic blood pressure (DBP), heart rate (HR), low-frequency (LF) power, $\mathrm{TH} / \mathrm{GAP} 43$ value, and LF/HF ratio were reduced to normal levels when MI rats were treated with NONRATT021972 siRNA. However, the decreased high-frequency (HF) power was increased. NONRATT021972 siRNA treatment also decreased the serum norepinephrine (NE) and epinephrine (EPI) concentrations in the MI rats [34]. Meanwhile, the increased $\mathrm{P}_{2} \mathrm{X}_{7} \mathrm{mRNA}$ and protein levels and the increased p-ERK1/2 expression in the SCG were also reduced. NONRATT021972 siRNA treatment inhibited the $\mathrm{P}_{2} \mathrm{X}_{7}$ agonist BzATP activated currents in HEK293 cells transfected with pEGFP-P2X7 [33]. These findings suggest that NONRATT021972 siRNA could decrease the upregulation of the $\mathrm{P}_{2} \mathrm{X}_{7}$ receptor and improve the abnormal changes in cardiac function after myocardial ischemia (Figure 2).

Study on MI rat suggested that treatment with NONRATT021972 siRNA, $\mathrm{P}_{2} \mathrm{X}_{7}$ siRNA or the $\mathrm{P}_{2} \mathrm{X}_{7}$ antagonist brilliant blue $G$ (BBG) enhanced the histology of wounded ischemic heart tissues and reduced the elevated concentrations of serum myocardial enzymes, CK, CK-MB, LDH and AST in MI rats. The underlying mechanism of p38 MAPK in myocardial ischemic injury is still not clear, so further study is needed. Compared to MI rats, there was a decrease in the expression level of $\mathrm{P}_{2} \mathrm{X}_{7}$ immunoreactivity, $\mathrm{P} 2 \mathrm{X}_{7} \mathrm{mRNA}$ and $\mathrm{P} 2 \mathrm{X}_{7}$ protein, IL-6, TNF-a, and phosphorylated p38 MAPK in the SG of MI rats treated with NONRATT021972 siRNA [34]. These findings suggest that NONRATT021972 siRNA treatment prevented the pathophysiologic processes mediated by $\mathrm{P}_{2} \mathrm{X}_{7}$ receptors in the SG after myocardial ischemic injury (Figure 2). Thus, the cardiac function after myocardial ischemia is improved when a subject is treated with NONRATT021972 siRNA indicating that NONRATT021972 may be a therapeutic target in MI.

\section{Sympathetic neuronal injury induced by ischemia}

High pressure, stretching or cut are the main causes for nerve injury. Sympathetic neuronal injury is the injury of the sympathetic nervous system whose main function is to stimulate the body's fight-or-flight response. Myocardial ischemia may lead to nerve injury [35]. LncRNA plays an important role on both normal development and diseases of the nervous system [36, 37]. NONRATT021972 expression levels were increased in PC12 cells subjected to oxygen-glucose deprivation (OGD) than those in cells cultured in control medium [36]. Thus, the NONRATT021972 lncRNA may relay to the pathophysiological changes of PC12 neuronal injury induced by OGD [36].

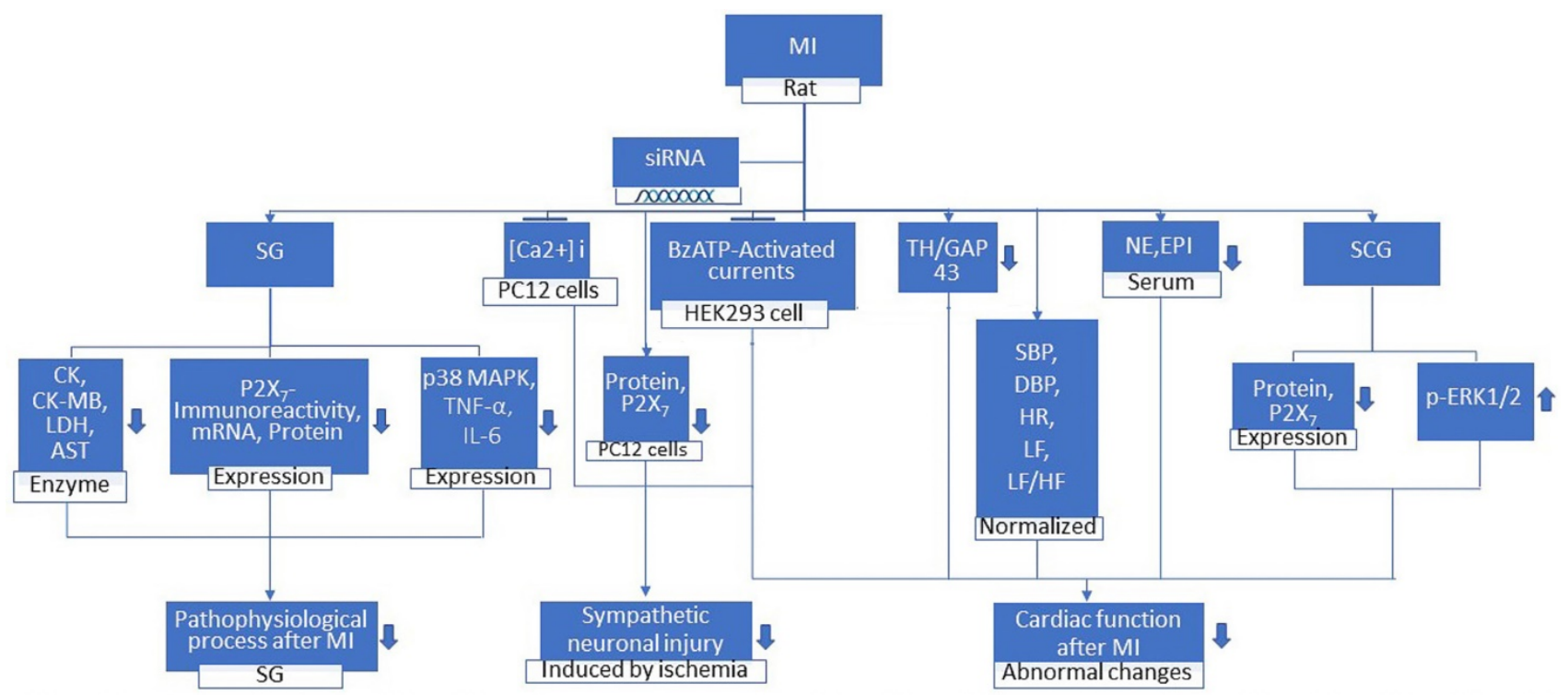

Figure 2. MI Rat, when treated with siRNA, leads to the decrease in ck, ck-mb, LDH, AST enzymes, serum Epinephrine and Norepinephrine and the expression of P2X immunoreactivity, mRNA, protein, p38 MAPK, TNF-a, IL-6, TH/GAP43. Whereas the p-ERK1/2 expression in superior cervical ganglia (SCG) increases. It also leads to the inhibition of $\left[\mathrm{Ca}^{2+}\right]$ i in oxygen and glucose deprived PC12 cells and BzATP-activated current in HEK293 cell. The systolic and diastolic blood pressure, heart rate, low-frequency (LF) power, and LF/HF ratio are normalized. These changes result in the prevention and reduction in pathophysiological processes mediated in stellate ganglia (SG) after Myocardial Infarction (MI), sympathetic neuronal injury induced by ischemia and abnormal changes in cardiac function after MI. 
NONRATT021972 siRNA treatment reversed the viability effect of OGD in PC12 cells. Studies showed that the expression levels of $\mathrm{P}_{2} \mathrm{X}_{7}$ mRNA as well as protein were significantly elevated after PC12 cells that were subjected to OGD were exposed to a selective ${\mathrm{P} 2 X_{7}}_{7}$ agonist (BzATP). NONRATT021972 siRNA treatment decreased the overexpression levels of $\mathrm{P}_{2} \mathrm{X}_{7} \mathrm{mRNA}$ and protein in PC12 cells. Treatment with NONRATT021972 siRNA in PC12 cells may reduce $\mathrm{P} 2 \mathrm{X}_{7}$ expression and prevent the sympathetic neuronal damage in OGD pathological conditions [36]. $\left[\mathrm{Ca}^{2+}\right] \mathrm{i}$ concentration in PC12 cells after OGD was inhibited after NONRATT021972 siRNA treatment. Li et al. suggested that treatment with NONRATT021972 siRNA may decrease the overactivation of the $\mathrm{P} 2 \mathrm{X}_{7}$ receptor and attenuate the sympathetic neuronal injury after exposure to OGD [36]. It is possible that after the exposure to OGD, NONRATT021972 may be involved in the abnormal changes of cell viability and $\left[\mathrm{Ca}^{2+}\right]$ i peak mediated by the $\mathrm{P} 2 \mathrm{X}_{7}$ receptor in $\mathrm{PC} 12$ cells. Silencing NONRATT021972 can decrease the sympathetic neuronal injury after exposure to OGD. Results showed that the inhibition of upregulation of the $\mathrm{P}_{2} \mathrm{X}_{7}$ receptor induced by OGD in PC12 cells can occur due to NONRATT021972 siRNA treatment which may reduce the $\mathrm{P}_{2} \mathrm{X}_{7}$ receptor-mediated neuronal injury induced by OGD. Therefore, it is urgent to find specific and sensitive biomarker for neuronal injury to improve clinical outcomes. The NONRATT021972 lncRNA may participate in the pathophysiological process of PC12 neuronal injury induced by OGD. There was an improvement in the pathological changes of the cell viability and $\left[\mathrm{Ca}^{2+}\right] \mathrm{i}$ peaks affected by OGD and prevention of the sympathetic neuronal injury following ischemia after treatment with NONRATT021972 siRNA [36] (Figure 2). As a conclusion, NONRATT021972 may be a valuable marker for systemic neuronal injury induced by ischemia.

\section{Hepatic glucokinase in T2DM}

In association with diabetes, glucokinase (GK) gene was the first gene to be identified [38]. Glucokinase (hexokinase IV or D) catalyzes the phosphorylation of glucose to glucose-6-phosphate (G6P) in parenchymal hepatocytes in the liver and in glucose-sensory endocrine and neuroendocrine cells within the pancreatic islets, gut, and brain involved in the control of blood glucose homeostasis [39]. The function of hepatic GK is to facilitate effective hepatic extraction of glucose postprandially [40]. In blood glucose homeostasis, the function of liver involves production of glucose in post absorptive state and extraction of glucose in postprandial state [41]. Controlling blood glucose levels and maintaining cellular metabolic functions are the essential role that is played by Hepatic GK [42, 43]. In T2DM, the expression of Hepatic GK and its activity are decreased [44]. Hepatic GK expression and glycogen synthesis are increased by the activation of protein kinase B (PKB/AKT) [45]. GK synthesis is decreased by the activation of glycogen synthase kinase-3 (GSK-3) and contributes to dysfunctional glucose metabolism [46].

LncRNA contributes in endocrine functions and diseases [47]. It has been reported that lncRNAs show potential as direct targets for therapeutic intervention in hepatic diseases [48]. Study done by Song et al. showed that the expression of NONRATT021972 in T2DM rat livers was higher than in control livers [46]. These results suggest that NONRATT021972 contributes in pathological and physiological processes in T2DM livers and disease development [46]. Studies demonstrated that the levels of Fasting Blood Glucose (FBG) and fasting plasma insulin (FINS) in the type 2 diabetic rats treated with saline group were higher than those in the normal saline control group. These results indicated that there was increment in the FBG and FINS levels of T2DM rats. Compared with the T2DM model rats, there was a significant improvement in the levels of FBG and FINS of T2DM rats that were treated with NONRATT021972 siRNA. The data suggested that significant normalization of abnormal FBG and FINS can be achieved by NONRATT021972 siRNA treatment. The levels of hepatic glycogen in T2DM rats and hepatocytes cultured with high glucose and fatty acids were significantly lower than those in control rats and hepatocytes. These results specified that hepatic glycogen synthesis was decreased in T2DM rats. Results showed that the expression level of GK mRNA and protein in control rats and hepatocytes were much higher than those in T2DM and hepatocytes cultured with high glucose and fatty acids [46]. Control of blood glucose level and the maintenance of cellular metabolic function is regulated by Hepatic GK [42]. By upregulating the expression of both p-AKT1 and GK and downregulating the phosphorylation and activation of GSK-3 $\beta$, treatment with NONRATT021972 helps in normalizing the levels of FBG and FINS to increase hepatic glycogen synthesis and reduce FBG levels [46] (Figure 3). Though the precise underlying mechanisms are yet to be elucidated, HGK and LncRNA NONRATT021972 may be researched as a future therapeutic approach for the treatment of T2DM. In conclusion, NONRATT021972 siRNA treatment may have beneficial effects on diabetes through hepatic glucokinase. 


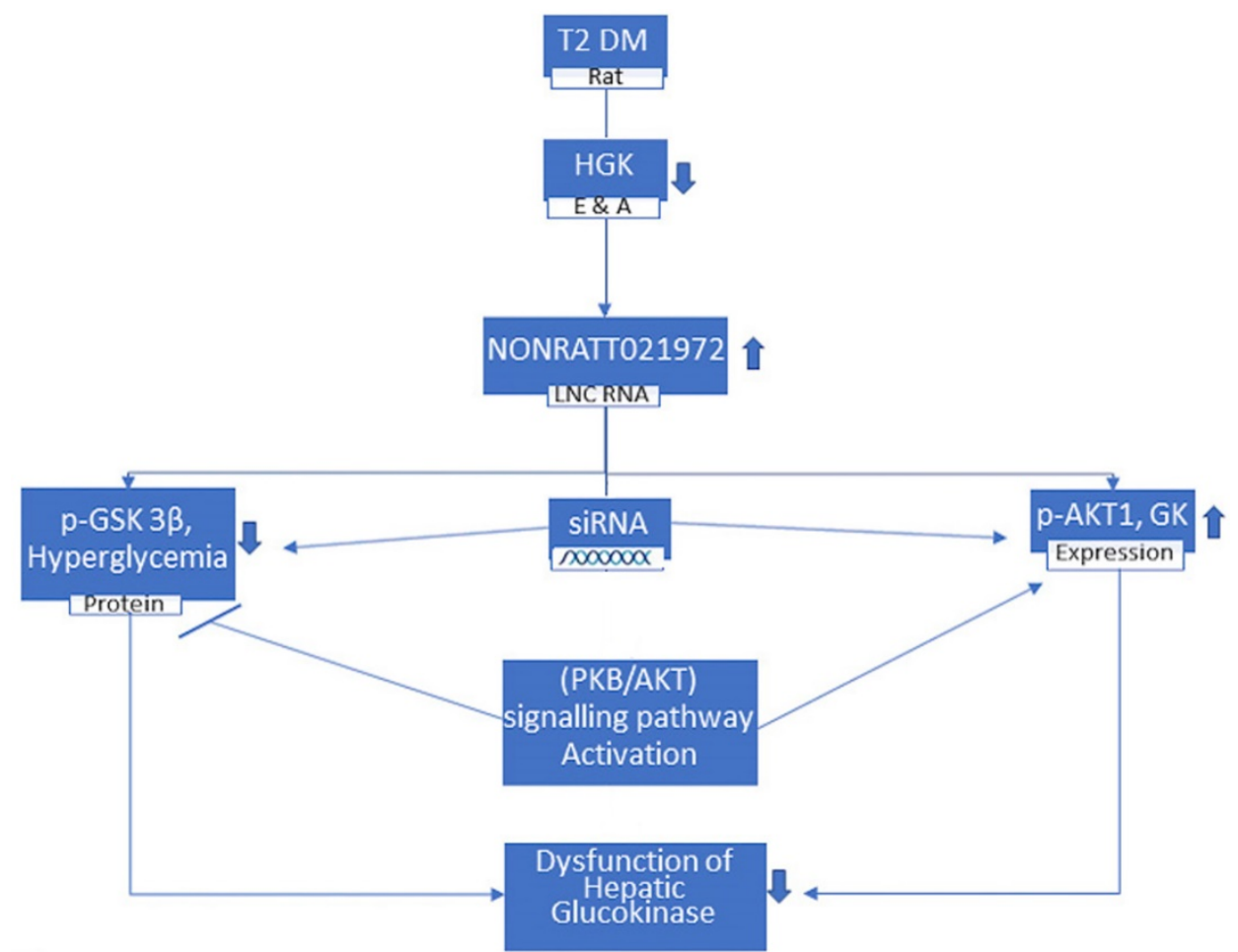

Figure 3. In T2 DM rats, where the Hepatic Glucokinase (HGK) is lower, the level of IncRNA NONRATT021972 is increased. These rats, when treated with siRNA NONRATT021972, leads to decrease in the P-GSK $3 \beta$ protein and reduction in hyperglycemia whereas increase in $\mathrm{p}$-AKT1 and Glucokinase (GK) expression. These are regulated by (PKB/AKT) signaling pathway activation and results in the improvement in the dysfunction of HGK.

\section{Conclusion and Future Aspects}

As we can see that the treatment with lncRNA NONRATT021972 siRNA in various conditions helps in relieving the symptoms of that specific disease. Neuropathic pain scoring was positively associated with lncRNA NONRATT021972 in patients with type 2 diabetes. It was also discovered to be a novel and efficacious link for DCAN. Prevention of the pathophysiologic processes mediated by $\mathrm{P} \mathrm{X}_{7}$ receptors in the SG after myocardial ischemic injury and improvement in abnormal changes in cardiac function after myocardial ischemia were possible by its treatment. NONRATT021972 siRNA also improved the pathological changes of the cell viability and prohibited the sympathetic neuronal injury ensuing ischemia. Normalization of the dysfunction of hepatic glucokinase through AKT signalling in T2DM rats was also achieved by the treatment of LncRNA NONRATT021972 siRNA. These all lead to a conclusion that the study of IncRNA NONRATT021972 shall provide vital information in the field of medicine about the complications of DM and cardiovascular disease. These findings highlight the potential of lncRNA NONRATT021972 as a novel therapeutic target in DNP. With the evolving treads in the medical science, lncRNA NONRATT021972, with current diagnostic technologies, could be used as the marker for the noxious stimuli as well as studying the response of the altered metabolic conditions. Its role as the therapeutic monitoring as well as therapeutic use could be explored for the welfare of the human beings.

\section{Abbreviation}

DRG: Dorsal Root Ganglia; TNF- $a$ : Tumor Necrosis Factor- $\alpha$; SCG: Superior Cervical Ganglion; siRNA: small interference RNA; SGC: Satellite Glial Cell; HRV: Heart rate variability; CAD: Coronary artery disease; HG: High glucose; HFs: High free fatty acids; IRS1: Insulin receptor substrate 1; MI: Myocardial Ischemia; SG: Stellate Ganglia; IL-6: Interleukin-6; p38 MAPK: p38 Mitogen Activated Protein Kinase; MAP: Mean arterial blood pressure; p-ERK1/2: Phosphorylated extracellular signalregulated protein kinases; HEK293: Human embryonic kidney cells 293; OGD: Oxygen - glucose deprivation; GK: Glucokinase; GSK-3: glycogen synthase kinase-3.

\section{Acknowledgements}

This work was supported by grants by the National Natural Science Foundation of China (Grant No. 81800828).

\section{Competing Interests}

The authors have declared that no competing interest exists. 


\section{References}

1. Vance KW, Ponting CP. Transcriptional regulatory functions of nuclear long noncoding RNAs. Trends Genet. 2014; 30: 348-55.

2. Knauss JL, Sun T. Regulatory mechanisms of long noncoding RNAs in vertebrate central nervous system development and function. Neuroscience. 2013; 235: 200-14.

3. Ponting $\mathrm{CP}$, Belgard TG. Transcribed dark matter: meaning or myth? Hum Mol Genet. 2010; 19: R162-8.

4. Pastori C, Wahlestedt C. Involvement of long noncoding RNAs in diseases affecting the central nervous system. RNA Biol. 2012; 9: 860-70.

5. Chen D, Sun Q, Cheng X, Zhang L, Song W, Zhou D, et al. Genome-wide analysis of long noncoding RNA (lncRNA) expression in colorectal cancer tissues from patients with liver metastasis. Cancer Med. 2016; 5: 1629-39.

6. Sauvageau M, Goff LA, Lodato S, Bonev B, Groff AF, Gerhardinger C, et al. Multiple knockout mouse models reveal lincRNAs are required for life and brain development. Elife. 2013; 2: e01749.

7. Sallam T, Sandhu J, Tontonoz P. Long Noncoding RNA Discovery in Cardiovascular Disease: Decoding Form to Function. Circ Res. 2018; 122: $155-66$.

8. Collaboration NCDRF. Worldwide trends in diabetes since 1980: a pooled analysis of 751 population-based studies with 4.4 million participants. Lancet. 2016; 387: 1513-30.

9. Xu Y, Wang L, He J, Bi Y, Li M, Wang T, et al. Prevalence and control of diabetes in Chinese adults. JAMA. 2013; 310: 948-59.

10. Callaghan BC, Cheng HT, Stables CL, Smith AL, Feldman EL. Diabetic neuropathy: clinical manifestations and current treatments. Lancet Neurol. 2012; 11: 521-34

11. Wang $\mathrm{M}$, Yao $\mathrm{D}$, Wang $\mathrm{S}$, Yan $\mathrm{Q}$, Lu W. Long non-coding RNA ENSMUST00000147869 protects mesangial cells from proliferation and fibrosis induced by diabetic nephropathy. Endocrine. 2016; 54: 81-92.

12. Wang M, Wang $\mathrm{S}$, Yao $\mathrm{D}, \mathrm{Yan} \mathrm{Q}, \mathrm{Lu} \mathrm{W}$. A novel long non-coding RNA CYP4B1-PS1-001 regulates proliferation and fibrosis in diabetic nephropathy. Mol Cell Endocrinol. 2016; 426: 136-45.

13. Yu W, Zhao GQ, Cao RJ, Zhu ZH, Li K. LncRNA NONRATT021972 Was Associated with Neuropathic Pain Scoring in Patients with Type 2 Diabetes. Behav Neurol. 2017; 2017: 2941297.

14. Yu Y, Fuscoe JC, Zhao C, Guo C, Jia M, Qing T, et al. A rat RNA-Seq transcriptomic BodyMap across 11 organs and 4 developmental stages. Nat Commun. 2014; 5: 3230.

15. Batista PJ, Chang HY. Long noncoding RNAs: cellular address codes in development and disease. Cell. 2013; 152: 1298-307.

16. Wapinski O, Chang HY. Long noncoding RNAs and human disease. Trends Cell Biol. 2011; 21: 354-61.

17. Li Z, Li X, Chen X, Li S, Ho IHT, Liu X, et al. Emerging roles of long non-coding RNAs in neuropathic pain. Cell Prolif. 2018: e12528.

18. Xu H, Liu C, Rao S, He L, Zhang T, Sun S, et al. LncRNA NONRATT021972 siRNA rescued decreased heart rate variability in diabetic rats in superior cervical ganglia. Auton Neurosci. 2016; 201: 1-7.

19. Liu S, Zou L, Xie J, Xie W, Wen S, Xie Q, et al. LncRNA NONRATT021972 siRNA regulates neuropathic pain behaviors in type 2 diabetic rats through the P2X7 receptor in dorsal root ganglia. Mol Brain. 2016; 9: 44.

20. Zhou B, Yu JW. A novel identified circular RNA, circRNA_010567, promotes myocardial fibrosis via suppressing miR-141 by targeting TGF-beta1. Biochem Biophys Res Commun. 2017; 487: 769-75.

21. Xu H, He L, Liu C, Tang L, Xu Y, Xiong M, et al. LncRNA NONRATT021972 siRNA attenuates P2X7 receptor expression and inflammatory cytokine production induced by combined high glucose and free fatty acids in PC12 cells. Purinergic signalling. 2016; 12: 259-68.

22. Katona I, Weis J. Diseases of the peripheral nerves. Handb Clin Neurol. 2017; 145: 453-74.

23. Peng H, Zou L, Xie J, Wu H, Wu B, Zhu G, et al. IncRNA NONRATT021972 siRNA Decreases Diabetic Neuropathic Pain Mediated by the P2X3 Receptor in Dorsal Root Ganglia. Mol Neurobiol. 2017; 54: 511-23.

24. Burnstock G. Purinergic signalling: from discovery to current developments. Exp Physiol. 2014; 99: 16-34.

25. Standl E, Schnell O. A new look at the heart in diabetes mellitus: from ailing to failing. Diabetologia. 2000; 43: 1455-69.

26. Bissinger A. Cardiac Autonomic Neuropathy: Why Should Cardiologists Care about That? J Diabetes Res. 2017; 2017: 5374176.

27. Martin-Timon I, Sevillano-Collantes C, Segura-Galindo A, Del Canizo-Gomez FJ. Type 2 diabetes and cardiovascular disease: Have all risk factors the same strength? World J Diabetes. 2014; 5: 444-70.

28. Armour JA. Potential clinical relevance of the 'little brain' on the mammalian heart. Exp Physiol. 2008; 93: 165-76.

29. Ferdinandy P, Schulz R, Baxter GF. Interaction of cardiovascular risk factors with myocardial ischemia/reperfusion injury, preconditioning, and postconditioning. Pharmacol Rev. 2007; 59: 418-58.

30. Rawshani A, Sattar N, Franzen S, Rawshani A, Hattersley AT, Svensson AM, et al. Excess mortality and cardiovascular disease in young adults with type 1 diabetes in relation to age at onset: a nationwide, register-based cohort study. Lancet. 2018; 392: 477-86.

31. Gao S, Zhan L, Yang Z, Shi R, Li H, Xia Z, et al. Remote Limb Ischaemic Postconditioning Protects Against Myocardial Ischaemia/Reperfusion Injury in Mice: Activation of JAK/STAT3-Mediated Nrf2-Antioxidant Signalling. Cell Physiol Biochem. 2017; 43: 1140-51.

32. Nanayakkara S, Marwick TH, Kaye DM. The ageing heart: the systemic and coronary circulation. Heart. 2018; 104: 370-6.

33. Tu G, Zou L, Liu S, Wu B, Lv Q, Wang S, et al. Long noncoding NONRATT021972 siRNA normalized abnormal sympathetic activity mediated by the upregulation of $\mathrm{P} 2 \mathrm{X} 7$ receptor in superior cervical ganglia after myocardial ischemia. Purinergic signalling. 2016; 12: 521-35.

34. Zou L, Tu G, Xie W, Wen S, Xie Q, Liu S, et al. LncRNA NONRATT021972 involved the pathophysiologic processes mediated by P2X7 receptors in stellate ganglia after myocardial ischemic injury. Purinergic signalling. 2016; 12: $127-37$.

35. Kong F, Liu S, Xu C, Liu J, Li G, Li G, et al. Electrophysiological studies of upregulated P2X7 receptors in rat superior cervical ganglia after myocardial ischemic injury. Neurochem Int. 2013; 63: 230-7.

36. Li G, Zou L, Xie W, Wen S, Xie Q, Gao Y, et al. The effects of NONRATT021972 IncRNA siRNA on PC12 neuronal injury mediated by P2X7 receptor after exposure to oxygen-glucose deprivation. Purinergic Signal. 2016; 12: 479-87.

37. Wang KC, Chang HY. Molecular mechanisms of long noncoding RNAs. Mol Cell. 2011; 43: 904-14.

38. Agius L. Hormonal and Metabolite Regulation of Hepatic Glucokinase. Annu Rev Nutr. 2016; 36: 389-415.

39. Matschinsky FM, Magnuson MA, Zelent D, Jetton TL, Doliba N, Han Y, et al. The network of glucokinase-expressing cells in glucose homeostasis and the potential of glucokinase activators for diabetes therapy. Diabetes. 2006; 55: $1-12$.

40. Agius L. Glucokinase and molecular aspects of liver glycogen metabolism. Biochem J. 2008; 414: 1-18.

41. Moore MC, Coate KC, Winnick JJ, An Z, Cherrington AD. Regulation of hepatic glucose uptake and storage in vivo. Adv Nutr. 2012; 3: 286-94.

42. Matschinsky FM. Assessing the potential of glucokinase activators in diabetes therapy. Nat Rev Drug Discov. 2009; 8: 399-416.

43. Iynedjian PB. Molecular physiology of mammalian glucokinase. Cell Mol Life Sci. 2009; 66: 27-42

44. Haeusler RA, Camastra S, Astiarraga B, Nannipieri M, Anselmino M, Ferrannini E. Decreased expression of hepatic glucokinase in type 2 diabetes. Mol Metab. 2015; 4: 222-6.

45. Xie X, Li W, Lan T, Liu W, Peng J, Huang K, et al. Berberine ameliorates hyperglycemia in alloxan-induced diabetic C57BL/ 6 mice through activation of Akt signaling pathway. Endocr J. 2011; 58: 761-8.

46. Song M, Zou L, Peng L, Liu S, Wu B, Yi Z, et al. LncRNA NONRATT021972 siRNA normalized the dysfunction of hepatic glucokinase through AKT signaling in T2DM rats. Endocr Res. 2017; 42: 180-90.

47. Sun M, Kraus WL. Minireview: Long noncoding RNAs: new "links" between gene expression and cellular outcomes in endocrinology. Mol Endocrinol. 2013; 27: 1390-402

48. Takahashi K, Yan I, Haga H, Patel T. Long noncoding RNA in liver diseases. Hepatology. 2014; 60: 744-53. 